\title{
Hikikomori silent epidemic: a case study
}

\author{
Ante Silić, ${ }^{1}$ Jakša Vukojević, ${ }^{2}$ Ilaria Čulo, ${ }^{2}$ Hrvoje Falak ${ }^{3}$ \\ ${ }^{1}$ Department of Psychiatry, Sestre Milosrdnice University Hospital Center, Zagreb; ${ }^{2}$ Department for Urgent Psychiatry, Vrapče University \\ Psychiatric Hospital, Zagreb; ${ }^{3}$ Department of Cardiology, Dubrava University Clinical Hospital, Zagreb, Croatia
}

\begin{abstract}
Psychiatry, as we know it, is at a crucial point because it needs to adapt to the modern time and still maintain the integrity and ethic aspects of the therapeutic alliance. Bearing in mind the rising prevalence of new addictions like Internet and online gaming addictions, one can see that, however, disputed, there is a whole new category of psychiatric illnesses on the rise. An example of these kinds of illnesses is Hikikomori. Hikikomori, or severe social withdrawal, pertains to patients who have stopped participating in everyday routine and would spend the majority of time confined in their room for the period of 6 months or more, with no evident psychosis. Although this syndrome was originally described in Japan, over the course of last few years it has been documented in several parts of the world, spreading like a silent epidemic. Our case study, being the first documented case in Southeast Europe, according to our experience and literature search, is a vivid example of this syndrome. In this report we discuss differential diagnosis, show what kind of therapy was efficient in the successful treatment of this syndrome and how it can be prevented in the future.
\end{abstract}

Key words: Hikikomori; Social withdrawal; Trauma; Internet; Psychosis; Psychotherapy.

Correspondence: Ante Silić, Department of Psychiatry, Sestre Milosrdnice University Hospital Center, Vinogradska cesta 29, 10000 Zagreb, Croatia.

E-mail: ante.silic@gmail.com

Citation: Silić, A., Vukojević, J., Čulo, I., \& Falak, H. (2019). Hikikomori silent epidemic: a case study. Research in Psychotherapy: Psychopathology, Process and Outcome, 22(2), 317-322. doi: 10.4081/ripppo.2019.377

Contributions: AS made substantial contributions to the conception and design of the work as well as analysis and interpretation of data for the work and revised the manuscript for important intellectual content; JV made substantial contributions to the conception and design of the work as well as analysis and interpretation of data for the work and drafted the manuscript; IC made substantial contributions to interpretation of data for the work and drafted the manuscript; HF contributed to the conception and design of the work as well acquisition and interpretation of data for the work and revised the manuscript. All authors gave final approval of the version to be published; they are accountable for all aspects of the work in ensuring that questions related to the accuracy or integrity of any part of the work are appropriately investigated and resolved.

Conflict of interest: the authors declare no potential conflict of interest.

Funding: none.

Received for publication: 20 January 2019

Revision received: 15 March 2019.

Accepted for publication: 26 April 2019.

This work is licensed under a Creative Commons Attribution NonCommercial 4.0 License (CC BY-NC 4.0).

${ }^{\circ}$ Copyright: the Author(s), 2019

Licensee PAGEPress, Italy

Research in Psychotherapy:

Psychopathology, Process and Outcome 2019; 22:317-322

doi:10.4081/ripppo.2019.377

\section{Introduction}

As psychiatry enters a new age, it is confronted with new and continuously developing psychopathology as we speak. An emerging problem in today psychiatry is social isolation. Although this problem is not new for psychiatrists, the psychopathology of social isolation has changed and therefore the way we perceive it. Today social isolation is becoming increasingly evident and is causing problems in several aspects, ranging from one's personal health to macroeconomy problems (Ministry of Health, 2003). That is even more prominent in the context of the increasing prevalence of new addictions such as addiction to gaming or Internet with prevalence estimating from less than $1.0 \%$ to more than $26.8 \%$ (Sussman, Sussman, Harper, Stahl, \& Weigle, 2018). The possibility to have a life outside of the real world is tempting for many, as it presents itself as an escape from the problems that are burdening them in real life. Some individuals become engulfed by the virtual world to extremes and thereby cut all ties with real life. All of the above mentioned is an emerging problem but however novel and sometimes disputed, it is increasingly evident that there is an entirely new category of psychiatric illnesses on the rise (Bhugra et al., 2017).

An illness that falls in the field of previously described problems is Hikikomori or severe youth social withdrawal. Hikikomori syndrome was initially described in Japan and, based on several case reports. It was initially defined as a form of social withdrawal in which those who are completely isolated from society and are in their home for more than six months, beginning in the second half of the twenties, and in which other psychiatric disorders do not 
better explain the main causes of this condition (Saito, 1998). At first, it was regarded as a social phenomenon endemic to Japan with a prevalence of $1.2 \%$ (Kato, Kanba, $\&$ Teo, 2018). In the next 20 years, Hikikomori cases started to emerge all over the world and are captivating the attention of clinician's day by day (Hayakawa et al., 2018), suggesting that the disorder is not at all endemic to Japan, and is transcending initial cultural boundaries (Harding, 2018). Until recently, all of the clinical knowledge was scares and mostly comprised of case reports, which was the main reason why there were no clear definitions and no criteria proposed. Information gathered through many case reports suggested that there are a few distinctive features that are associated with Hikikomori (Stip, Thibault, Beauchamp-Chatel, \& Kisely, 2016; Teo et al., 2015). Many cases described that Hikikomori patients had been victims of bullying or other forms of peer rejection (Lee, Lee, Choi, \& Choi, 2013), had dysfunctional family dynamics (parental rejection or overprotection) (Stip et al., 2016) and were at risk of Internet addiction (Lee et al., 2013). Furthermore, gathered psychosocial data suggested that the average Hikikomori patient was lonely and deficient in social support, although he does not live alone, and is unable to maintain meaningful relationships (Teo et al., 2015). In 2015, a small study ( $\mathrm{n}=24)$ focused on the relationship between attachment style and Hikikomori syndrome, concluded that ambivalent attachment, as a result of parental rejection, in combination with peer rejection significantly predicts Hikikomori, while temperamental shyness can additionally influence Hikikomori (Krieg \& Dickie, 2013). The first and only 12month study on Hikikomori was designed and, although the sample was small $(n=190)$, it was the largest sample size to date and yielded interesting clinical information (Malagón-Amor et al., 2018). The study identified that an intensive treatment, such as hospitalization, combined with psychopharmachotherapy, psychotherapy (individual and group), and socially oriented interventions gave the best outcomes (Malagón-Amor et al., 2018). Surprisingly faceto-face psychotherapy, as well as group therapy was preferred over Internet-based psychotherapy, suggesting that a multi-disciplinary (i.e., psychiatrist, psychotherapist, group therapist, social worker, occupational therapist) approach would give most beneficial effects (MalagónAmor et al., 2018). These results, regarding the therapy options and preferences, are the only that have clinical evidence although the same approach was hypothesized before (Harding, 2018; Krieg \& Dickie, 2013; Teo et al., 2015). Today the diagnostic criteria for Hikikomori are the following: i) spending most of the day and most days at home; ii) avoiding social situations such as attending school or going to work; iii) avoiding social relationships such as friendships or contacts with family members; iv) experiencing discomfort or significant deterioration due to social isolation; v) having a minimum duration of 6 months (Malagón-Amor et al., 2018; Teo \& Gaw, 2010).
At first glance, Hikikomori may resemble other serious psychiatric conditions like the prodromal phase of schizophrenia, or other psychotic disorders, and even major depressive disorder (MDD) but is relatively easy to differentiate from those two. Having no cognitive decline, no behavioral oddities and lack of other negative symptoms are the exclusion criteria for schizophrenia, while the absence of fatigue, appetite alterations, cognitive decline, and suicidal ideation exclude MDD as the underlying condition (Stip et al., 2016). Recently, Teo et al. (2018) developed a Hikikomori Questionnaire (HQ25) containing 25 items, across three subscales representing socialization, isolation, and emotional support, to help in the evaluation of a relatively new mental health issue that is emerging and to further distinguish this disorder from others with similar symptoms. All of the above mentioned points out to a new disorder that is developing, the disorder that resembles schizophrenia spectrum psychosis but on the other hand, it is closely intertwined with one's personality and development. Furthermore, a disorder that is closely related to new technologies, in a sense that Internet use and gaming can easily induce and subsequently protract the social isolation (Stip et al., 2016), and as such is only prone to develop into more severe psychopathology as time and technology progress.

\section{Aims and hypothesis}

The objective of this case study is to summon our therapeutic experience and relevant literature in order to present our therapeutic process and outcome regarding the treatment of Hikikomori syndrome. We hypothesize that Hikikomori is an emerging issue that needs to be tackled with a multidisciplinary approach and needs to be assessed as a biological but also a psychodynamic problem that cannot be treated solely with medication or psychotherapy, and as such a complex syndrome, it should be treated with a bio-psycho-social approach.

\section{Methods}

\section{Participant}

Here we present the case of a young male who was admitted to Vrapče University Psychiatric Hospital on September $17^{\text {th }}, 2017$. At the time he was 24 years old, lived with his parents and two siblings, an older brother, and younger sister. His birth and early psychomotor development were normal, and he showed no abnormalities. He describes his early childhood as pleasant, and his early memories are of playing with his siblings and being carefree. He sees his mother as caring but overprotective and strict while he has little to say about his father. When talking about his brother he describes him as not interested and distant as he grew up, while his sister was the one with whom he spent 
most of his time. He repeatedly compared his sister to his mother regarding their personalities. In preschool and elementary school, he had friends and had almost no bad memories from that period. He started experiencing first problems at the end of elementary school when he developed speech impairment. Although he has not had any obvious trauma, he started to stutter in his early teens. Right about that time he enrolled in high school and had difficulties socially adjusting to the new environment. He reports other pupils teasing him and making fun of him to the point of bullying. He describes his feeling towards school as hell on earth and uses only derogatory vocabulary. As a result, he thought of himself as less worthy and insignificant, which led to the development of a defeatist worldview, where people have bad intentions and are not to be trusted. Gradually he started to spend more and more time in isolation, staying at home and playing video games. Although he finished high school, he never attempted to enroll in college but continued to stay at home and avoid any social contact. For two years, he spent most of his day in the room, more than 20 hours a day, occasionally going out to keep his hygiene or to go to the toilet but the remainder of the time he was playing video games and excessively surfing the web. He was frequently confronted about his behavior by his parents, which resulted in aggressive outbursts and further isolation. At one point, he blocked his room door with furniture from inside of the room to stop family members from entering. Finally, his sister decided to call the ambulance to help him and to have him examined by professionals. Paramedics in ambulance noted that they were summoned due to patients' excessive gaming and isolation. He was brought to our emergency department accompanied by his father and paramedics. After the initial exam and subsequent admission to the hospital, his father left and was not present during his treatment process. All the information was given by his sister who told us that he has been spending most of the time in his room for a period of at least three years. He spent most of his time online, surfing the web and gaming, kept his hygiene, exercised regularly, ate and drank in his room. Psychiatrist on call decided that the patient needed to be admitted and observed for at least 48 hours according to Croatian Mental Health Act (Croatian Ministry of Law, 2015). Within the first 48 hours, our patient gave his informed consent for the differential diagnostic procedure and treatment.

\section{Measures and procedure}

Upon admission, every patient goes through our routine differential diagnostic procedure that consists of an initial evaluation of the patient using Clinical Global Impression scale (CGI), Electroencephalography (EEG), Computed Tomography (CT), laboratory testing and psychological assessment consisting of several tests.
Throughout the treatment process, which included one month of intensive in-ward treatment and one month of day hospital, the patient was consistently monitored, and the clinical progression was evaluated. Last evaluation was done on a 6-month follow up.

The CGI scale is used to give an initial impression of the patient's psychopathology and mental state. The CGI scale is used once at the beginning of the treatment and before demission, as to evaluate the progress of the treatment and patient's mental state before going home.

EEG is the measurement of the ongoing electrical activity of the brain recorded non-invasively from electrodes on the scalp. As such EEG is typically used in clinical circumstances to distinguish epileptic seizures from psychogenic non-epileptic seizures but also syncopes and sub-cortical movement disorders, it is also used to differentiate organic encephalopathy or delirium from primary psychiatric syndromes such as catatonia (LaRoche \& Haider, 2018).

CT scan detects and precisely localizes the intracranial hematomas, tumors, brain contusions, edema and foreign bodies (Rincon, Gupta, \& Ptak, 2016). Due to its widespread availability, the speed of acquisition, and lack of contraindications it is the first-line modality in the screening of brain conditions.

Laboratory tests are used to exclude preexisting somatic conditions that could be the cause of mental illness. Laboratory tests include endocrinology, inflammatory and haematopoetic illness screening as well as the assessment of the liver and kidney function and the electrolyte status.

Our psychological evaluation consists of two major tests. The Wechsler Adult Intelligence Scale-IV (WAISIV; Wechsler, 2008) which is an Intelligence Quotient (IQ) test designed to measure intelligence and cognitive ability in adults. The Cronbach's alpha reliability coefficients, for subtests in the WAIS-IV ranging from .87 to .98 , it is one of the most used and standardized IQ tests (Wechsler, 2008).

The Index Profile of Emotions (PIE; Plutchik \& Kellerman, 1974) is a standard questionnaire for delving into the diversity of personality's dimensions. Using the questionnaire, it is possible to measure the expression of eight primary emotions, all through following prototypes of behavior: incorporation, uncontrollability, selfprotection, deprivation, antagonism, exploration, destruction, reproduction. The alpha of Cronbach is above 0.9 , in each of the above-mentioned subfields, it is considered reliable (Milenković \& Jovanović, 2016).

\section{Data analysis}

To evaluate the improvements regarding resocialization, daily functioning and patients' attitude towards computer use, as well as time spent online, we assessed and compared all reports from our multidisciplinary team across time. In this case study, we did not use any statistical analysis. 


\section{Results}

EEG scan was within physiological parameters, showing just a diffuse dysrhythmic EEG finding that is not characteristic for any psychiatric condition. CT brain scan showed no structural abnormalities, no preexisting trauma or bleeding, as well as no incipient organic changes. The psychological evaluation consisted of several psychological tests with the purpose to evaluate the patients cognitive and personality traits. Laboratory finding where all in the expected range except mean corpuscular hemoglobin concentration an aspartate aminotransferase that were slightly higher and lower uric acid (UA) values.

The WAIS-IV showed an overall IQ score of 105 points placing the patient's score in the average of Croatian population. PIE showed increased aggressivity and depression, with scores of 100 and 72, respectively setting the patient in high centile in comparison to the average population (Plutchnik \& Kellerman, 1974). The same test also showed low scores in sociability, the patient scored 7 , and in uncontrollability where the patient scored 4 , both of which placed him in the low ten centiles of average population (Plutchnik \& Kellerman, 1974). Our clinical psychologist, using the tests mentioned above and several others, concluded that there is a possible persistent accommodation difficulty within avoidant personality traits (fear of life), possibly caused by narcissistic injury in early childhood, and in similar situations can occasionally be triggered into psychotic levels of reaction.

We introduced pharmacological treatment with a goal to tackle leading symptoms that were troubling our patient. Those being a high level of anxiety and low level of adjustment to new environment, we started pharmacotherapy with low doses of olanzapine $(5 \mathrm{mg}$ in the evening) and low doses of lorazepam (1 $\mathrm{mg}$ in the morning and the evening). He responded well to applied pharmacotherapy.

Therapeutic response was evaluated by the involved therapeutic team and by CGI scales (Guy, 1976), with CGI score lowering from 5 on admission to 1 on demission. Simultaneously with pharmacotherapy, we introduced psychotherapy (individual and group psychotherapy, cognitive-behavioral therapy (CBT), metacognitive training, psychodynamic psychotherapy, psychoeducation) and socio-occupational therapy. His sister was involved in psychoeducation and family therapy. Inpatient period of treatment lasted for one month ending with transference to outpatient Day hospital treatment of similar duration. After Day Hospital period of treatment, he continued treatment as an outpatient. Pharmacological treatment was titrated according to clinical presentation and level of functioning. We reduced the dosage of olanzapine to $2.5 \mathrm{mg}$ per day and eventually we discontinued olanzapine. Lorazepam was also reduced from $2 \mathrm{mg}$ per day to $1 \mathrm{mg}$ per day and eventually, lorazepam was also discontinued. As clinical presentation did not suggest deeper endogenous process and patient clearly did not meet the criteria for diagnoses of schizophrenia spectrum and other psychotic disorders, bipolar and related disorders, depressive disorders, anxiety disorders, obsessive-compulsive disorder (OCD), personality disorders and post-traumatic stress disorder (PTSD), we have chosen the Hikikomori syndrome as best fitting diagnosis for this particular patient. In our therapeutic approach, after one month of hospital treatment, he was admitted in Day hospital, which offered an outpatient treatment which included seven patients suffering from a first psychotic episode, aged between 20 and 30 years. All the patients have previously been diagnosed with first psychotic episodes and were taking antipsychotic medication.

The therapeutic program was conducted by a multidisciplinary team consisting of psychiatrists, psychologists, social worker, and occupational therapist, whose clinical guidelines were based on inputs from psychopharmacotherapy, psychoeducation, CBT, individual and group psychodynamic therapy, group metacognitive training, and community therapy.

Although the approach is multidisciplinary, for each patient, we developed unique treatment strategies. In the case of Hikikomori, the emphasis was on insight and resocialization.

Psychoeducation is performed once weekly in a fixed time slot. Basic principles are CBT based, and we have cycles of themes consisting of 12 topics. So, one cycle lasts for 12 weeks. It is interactive, and family members are allowed. Patients and family members can be enrolled at any point in the cycle but are encouraged to stay for the entire cycle. Sometimes patients and or family members get homework. This has proven useful with the younger population as they have many unanswered questions regarding their condition that stem from Internet searches. With this information, our patient and his family have the know-how to deal with his condition, and he was much more motivated to participate in other therapy modalities. Cognitive behavioral therapy, while on the ward, had an emphasis on behavioral activation and socialization efforts, while the use of electronic devices was restricted due to the ward rules, which proved to help limit the time spent on computers. In the day hospital we proceeded with the same regime, the emphasis was on socializing and going out instead of using a computer, but with the addition of diaries with daily activities and selfmonitoring remarks with the idea of providing better insight into one's behavior but also daily functioning and routine. As a result of CBT, upon demission, when our patient started to have full access to the PC and his room, the use of a computer was agreed to be more rational and was used as a reward. The use was permitted after carrying out specified daily tasks or when doing workrelated duties, alongside which he was also encouraged 
to be more engaged in hobbies as a substitute for time, previously spent online. A licensed psychiatrist conducted metacognitive training in a group setting in which, using interactive methods, the goal of the training was to deepen the insight of our patients and give them a way to reevaluate their actions and thoughts. In the case of Hikikomori, it was beneficial as he managed to gain better insight into his actions, which combined with psychoeducation and CBT as well as psychotherapy resulted in behavioral changes in our patient and changes in his daily routine as well.

The above-mentioned individual psychotherapy and group psychotherapy based on psychoanalytical principles were administered regularly every week. Individual psychoanalytical psychotherapy sessions were held twice a week while group psychotherapy was held once a week, on the ward as well as in the daily hospital. The main aim of these methods was to improve the social functioning of our patient and to extend the degree of insight and awareness about his image regarding early and high school trauma he experienced.

In individual therapy sessions, the main aim was to establish transference, and since our patient was socially withdrawn it took some time for him to talk about himself openly, he usually spoke only on therapist's initiative answering questions in short sentences. There was a lack of spontaneity in treatment, and a significant amount of unpleasant silence was present which was contributed to resistance to therapy, as a defense mechanism. However, after three weeks of mostly silence, he started to talk about how he is unhappy with the current situation, showed he was angry with his family that brought him to therapy. We explored these feelings of anger, and it was evident that he was angry about feeling neglected and out of place in his home as well as school and other plains of social life. He had a deep sense of not belonging and inadequacy. In therapy he frequently described how playing video games made him feel alive it was a place where he felt safe and could take on a new identity and detach the point that the real world lost meaning and significance. By playing video games and spending time on the Internet, he was able to detach himself from any feelings that overwhelmed him as if the game world was a place where he could feed his narcissistic desires without fear of getting hurt.

Through day hospital treatment he showed significant improvement in daily functioning and social interactions with other patients as well as with members of his family, even gaining friends he kept seeing outside of the hospital. He had access to his PC, which he did not use excessively, as was agreed in his CBT sessions, and all improvements were maintained on the outpatient follow-up. The first follow up was after one month while the second was after three months. On both follow-ups, one of the family members was present and confirmed statements given by the patient.

\section{Discussion and Conclusions}

The differential diagnostic procedure was particularly complicated for this patient. With our clinical experience and according to patient clinical presentation, the first diagnosis that we pursued was an acute psychotic disorder. The available anamnestic and heteroanamnestic data was also suggesting acute psychotic disorder or schizophrenia. However, this patient did not meet the diagnostic criteria for schizophrenia spectrum and other psychotic disorders (quality and duration of symptoms do not meet criteria for any subtype of schizophrenia and schizophrenia spectrum disorders). As for schizoaffective, bipolar disorder and major depressive disorder, since the patient was not clinically depressed or manic at any point in time during treatment and before according to anamnestic and heteroanamnestic data, criteria for establishing such diagnosis could not be met. Same applies for anxiety disorders, OCD, personality disorders and PTSD (lack of pervasive patterns, lack of obsessive thoughts/behavior, intrusive memories, disrupted sleeping patterns or any key symptom that would allow us to establish any diagnosis according to ICD-10 and DSM5). This was confirmed by thorough psychological evaluation and reevaluation as well as with quick response to pharmaco-psychotherapy. In every day clinical practice, we do not meet very often a patient that cannot be precisely diagnosed by our multidisciplinary team is given enough time and diagnostic tools. This patient stands out in that matter particularly because he did express occasional/situational psychotic levels of anxiety and psychotic level of functionality that did respond well to antipsychotic and anxiolytic treatment. That fact inspired us to look further into the differential diagnostic procedure. Upon through literature review, which was conducted using PubMed and researching all papers published on the Hikikomori topic since it was introduced in 1998 (Saito, 1998), we concluded that Hikikomori is the best fitting diagnosis as it met all the proposed diagnostic criteria. In addition to the met diagnostic criteria, we also found that our patient had severe social isolation with heavy Internet and gaming use (Hayakawa et al., 2018; Lee et al., 2013), childhood trauma in the sense of middle and high school bullying (Kato et al., 2018; Tajan, 2017) and as Hayakawa et al. suggested, we evaluated his personality traits to be avoidant, and his UA levels were low (Hayakawa et al., 2018).

Limitation of this case study is the fact that in Croatia we do not have any experience with diagnostics and treatment of Hikikomori syndrome and that this study is based on one case and thereby is hard to generalize the outcomes in a broader population. Another limitation is that we treated this patient in our First psychosis ward as it was the best fit for him regarding his age and resocialization program, but he may have been better off in an Internet addiction or Personality ward. 
The psychotherapeutic approach, consisting of psychoeducation, CBT, individual and group psychodynamic therapy, and metacognitive training, combined with low doses of psychodrugs yielded the best results for this kind of psychopathology in our case. We evaluated the therapeutic process by the quality and quantity of prominent symptoms and by the rate of resocialization. The structure of program on the First psychosis ward (Ostojic, Čulo, Silić, Kos, \& Savić, 2018) and later in the First psychosis Day hospital suited our patient well, as the program was not too demanding but structured with clear boundaries. The question remains if we could have caught the patient earlier in the process by implementing novel approaches, such as mobile teams or online approach as some authors suggested (Lee et al., 2013; Vukojevic \& Silic, 2018), considering that psychiatry is in the crucial point and is (mal)adapting to modern times (Bhugra et al., 2017).

\section{References}

Bhugra, D., Tasman, A., Pathare, S., Priebe, S., Smith, S., Torous, J., ... First, M. B. (2017). The WPA-lancet psychiatrycommission on the future of psychiatry. The Lancet Psychiatry, 4(10), 775-818.

Croatian Ministry of Law (2015). Croatian Mental Health Act. Retrived from: https://narodne-novine.nn.hr/clanci/sluzbeni/ 2014_06_76_1414.html

Guy, W. (1976). Clinical Global Impressions. ECDEU Assessment Manual for Psychopharmacology. Rockville, MD: US Department of Health, Education, and Welfare. DHEW Publication No. ADM 76-338.

Harding, C. (2018). Hikikomori. The Lancet Psychiatry, 5(1), 28-29.

Hayakawa, K., Kato, T. A., Watabe, M., Teo, A. R., Horikawa, H., Kuwano, N., ... Sagata, N. (2018). Blood biomarkers of Hikikomori, a severe social withdrawal syndrome. Scientific Reports, 8(1), 2884.

Kato, T. A., Kanba, S., \& Teo, A. R. (2018). Hikikomori: experience in Japan and international relevance. World Psychiatry, 17(1), 105.

Krieg, A., \& Dickie, J. R. (2013). Attachment and hikikomori: a psychosocial developmental model. International Journal of Social Psychiatry, 59(1), 61-72.

LaRoche, S. M., \& Haider, H. A. (Eds.). (2018). Handbook of ICU EEG monitoring. New York: Springer Publishing Company.

Lee, Y. S., Lee, J. Y., Choi, T. Y., \& Choi, J. T. (2013). Home visitation program for detecting, evaluating and treating socially withdrawn youth in Korea. Psychiatry and Clinical Neurosciences, 67(4), 193-202.
Malagón-Amor, Á., Martín-López, L. M., Córcoles, D., González, A., Bellsolà, M., Teo, A. R., ... Bergé, D. (2018). A 12-month study of the hikikomori syndrome of social withdrawal: Clinical characterization and different subtypes proposal. Psychiatry Research, 270, 1039-1046.

Milenković, T., \& Jovanović, A. (2016). The impact of emotional characteristics in thechronicity of posttraumatic stress disorder. Psychiatria Danubina, 28(2), 164-169.

Ministry of Health, L. A. W. (2003). Shakaiteki Hikikomori Wo Meguru Tiiki Seisin Hoken Katudou No Guide-line [Guideline on Mental health activities in communities for social withdrawal]. Tokio, Japan: Ministry of Health.

Ostojić, D., Čulo, I., Silić, A., Kos, S., \& Savić, A. (2018). Integrated specialized early-course psychosis treatment services-University Psychiatric Hospital Vrapce model. Psychiatria Danubina, 30(Suppl 4), 208-215.

Plutchik, R., \& Kellerman, H. (1974). Emotion Profile Index. Los Angeles: Western Psychological Services.

Rincon, S., Gupta, R., \& Ptak, T. (2016). Imaging of head trauma. In J. C. Masdeu \& R. G. González (Eds.), Handbook of clinical neurology (Vol. 135, pp. 447-477). Amsterdam: Elsevier.

Saito, T. (1998). Shakaiteki Hikikomori: Owaranai Shishunki [Social withdrawal: a never ending adolescence]. Tokyo, Japan: PHP Kenkyujo.

Stip, E., Thibault, A., Beauchamp-Chatel, A., \& Kisely, S. (2016). Internet addiction, Hikikomori syndrome, and the prodromal phase of psychosis. Front Psychiatry, 3(7),6. doi: 10.3389/fpsyt.2016.00006.

Sussman, C. J., Harper, J. M., Stahl, J. L., \& Weigle, P. (2018). Internet and video game addictions: Diagnosis, epidemiology, and neurobiology. Child and Adolescent Psychiatric Clinics, 27(2), 307-326.

Tajan, N. (2017). Traumatic dimensions of hikikomori: A Foucauldian note. Asian Journal of Psychiatry, 27, 121-122.

Teo, A. R., Chen, J. I., Kubo, H., Katsuki, R., Sato-Kasai, M., Shimokawa, N., ... Kato, T. A. (2018). Development and validation of the 25-item Hikikomori Questionnaire (HQ25). Psychiatry and Clinical Neurosciences, 72(10), 780-788.

Teo, A. R., Fetters, M. D., Stufflebam, K., Tateno, M., Balhara, Y., Choi, T. Y., ... Kato, T. A. (2015). Identification of the hikikomori syndrome of social withdrawal: psychosocial features and treatment preferences in four countries. International Journal of Social Psychiatry, 61(1), 64-72.

Teo, A. R., \& Gaw, A. C. (2010). Hikikomori, a Japanese culture-bound syndrome of socialwithdrawal? A proposal for DSM-V. The Journal of Nervous and Mental Disease, 198(6), 444.

Vukojević, J., \& Silić, A. (2018). The future of psychiatry and the story of two worlds. Asian Journal of Psychiatry, 35, 3.

Wechsler, D. (2008). Wechsler Adult Intelligence Scale. San Antonio, TX: NCS Pearson. 